\title{
Les variations diastratiques du français au Grand-Duché de Luxembourg
}

\author{
Nicolas Lefrançois \\ Laboratoire EA-739 DIPRALANG, Université Paul Valéry - Montpellier III, \\ 6, rue Jean-Martin Tschoffen, 6700 Arlon, Belgique
}

\begin{abstract}
Résumé. Dans la triglossie luxembourgeoise - allemand, français, luxembourgeois - le français tient une place particulière puisque tant son statut que son expansion ont varié au cours de l'histoire récente du pays. Langue littéraire de l'élite à l'époque de l'indépendance en 1831 dans un pays germanophone le français connait un pic de diffusion sociale durant la deuxième moitié $d u X^{\mathrm{e}}$ siècle, suite à un fort besoin de main-d'œuvre issue de la Belgique et de la France voisines ainsi que de l'immigration principalement portugaise. Cette "démocratisation" du français s'accompagne alors d'une dévalorisation pour les Luxembourgeois remettant en cause son statut de langue officielle du Grand-Duché.
\end{abstract}

\begin{abstract}
Diastratic variations of French in the Grand Duchy of Luxembourg. In the Luxembourg triglossia German, French, Luxembourg - French holds a special place because both its status and its expansion have varied in the recent history of the country. Literary language of the elite at the time of independence in 1831 in a German-speaking country, French experienced a peak of social diffusion during the second half of the twentieth century, following a strong need for hand-Belgium and France, as well as mainly Portuguese immigration. This "democratization" of French was accompanied by a devaluation for Luxembourgers calling into question its status as an official language of the Grand Duchy.
\end{abstract}

\section{Introduction}

Dans la triglossie luxembourgeoise - allemand, français, luxembourgeois - le français tient une place particulière puisque tant son statut que son expansion ont varié au cours de l'histoire récente du petit État lorrain. Langue littéraire de l'élite à l'époque de l'accession à l'indépendance en 1831 mais sans implantation dans la population autochtone, le français connait un pic de diffusion sociale avec la première vague de migrants italiens de la fin du $\mathrm{XIX}^{\mathrm{e}}$ siècle. Confiné cependant à cette seule frange marginale de la population, il conserve sa valeur de variété de prestige auprès des natifs qui se considèrent avant tout comme des germanophones. L'essor économique du Luxembourg, durant la deuxième moitié du $\mathrm{XX}^{\mathrm{e}}$ siècle, suscite un besoin conséquent de main-d'œuvre qui se recrute en particulier auprès des pays francophones voisins, la Belgique et la France. Cette croissance relance également 
l'immigration économique romanophone, principalement en provenance du Portugal. Le français se diffuse alors plus largement dans les zones économiquement actives du Luxembourg, le Centre et le Sud principalement, au point de devenir une véritable lingua franca intercommunautaire et la première langue d'intégration des étrangers. Toutefois, cette «démocratisation» du français s'accompagne d'une dévalorisation pour les Luxembourgeois au point de remettre en cause son statut de langue officielle du GrandDuché.

La présente communication situe diachroniquement la sociolinguistique luxembourgeoise en fonction de son histoire récente et de sa démographie particulière. En s'appuyant sur 1' 'architecture de langue' développée par Leiv Flydal et Eugenio Coseriu, le statut du français au Luxembourg est ensuite analysé à travers ses variations diastratiques, fruits de l'histoire économique et migratoire du pays. L'étude est étayée par les témoignages de Luxembourgeois ou de résidents de longue date au Luxembourg faisant part de leurs propres représentations et de leur appropriation du français. Ces témoignages ont été collectés en 2015 et en 2017 à l'occasion de la rédaction d'un mémoire de master de recherche et d'une thèse de doctorat en sociolinguistique.

\section{Triglossie en diachronie}

Par une révolution populaire en 1830, la Belgique et le Luxembourg se séparent du Royaume des Provinces-Unies des Pays-Bas pour former deux États indépendants. Suite à une conférence qui se tient à Londres en 1831, le redécoupage territorial, devant définir les frontières communes des deux États, ampute en 1839 le Luxembourg de sa région ouest, francophone au profit de la Belgique. Le Luxembourg devient dès lors un pays uniquement germanophone (Berg et Weis, 2005 p. 11). L'historien du Luxembourg Gilbert Trausch, cité par C. Berg et C. Weis, affirme que « depuis 1839 il n’y a plus de Luxembourgeois ayant le français comme langue maternelle » (Berg et Weis, 2005, p. 34). Mais le français ne disparait pas totalement du pays. Il s'y maintient en tant que langue administrative et institutionnelle par l'arrêté royal du 22 février $1834^{1}$ qui dispose que les deux langues officielles du Grand-Duché sont le français et l'allemand. En réalité, le français doit sa présence légale à la volonté de la haute bourgeoisie locale, héritière des idées révolutionnaires importées dans le pays lors de l'invasion des troupes napoléoniennes en 1795. Napoléon y a imposé un Code pénal, un Code de commerce et un Code civil rédigés en français et qui satisfont les ambitions émancipatrices des élites locales face au pouvoir du roi des Pays-Bas, Grand-Duc du Luxembourg. Par ailleurs, le français appartient à l'histoire de ce petit pays aux confins de l'Empire Romain germanique et du Royaume de France. Outre une partie de la Wallonie francophone cédée en 1839 à la Belgique, le Luxembourg a vu ses frontières s'étendre sur une frange de la Lorraine et des Ardennes françaises jusqu'au milieu du XVIII ${ }^{\mathrm{e}}$ siècle et le français était alors langue de la chancellerie et de la Cour (Frisch, 1998, p. 108). Il n'est donc pas une variété importée ou imposée mais un parler endémique pour une partie de la population (Berg et Weis, 2005, p. 34). Enfin, «jusqu'à la Première Guerre mondiale une petite frange des couches supérieures de la bourgeoisie utilise, dans ses foyers, le français » (Trausch, 1992, p. 106).

nicolaslefrancois69@gmail.com 
Cette même bourgeoisie a maintenu l'usage du français comme langue de l'administration et de la culture à côté de l'allemand, sans pour autant accorder la préséance à cette dernière malgré son importance et sa diffusion locales par défiance vis-à-vis du puissant voisin prussien (ibid.). A sa naissance, le Luxembourg éclot donc dans une configuration officiellement bilingue français-allemand. Mais l'allemand lui-même se divise en deux variétés : une variété littéraire et scripturaire, le haut allemand (Hochdeutesch) et une variété régionale vernaculaire, l'allemand luxembourgeois (lëtzbuerger-daitsch) (Trausch, 1992, p. 109), ancêtre du futur luxembourgeois officialisé institutionnellement en 1984.

En 1839, le Luxembourg est un pays pauvre et rural qui voit sa population fuir à l'étranger et principalement vers la France et les États-Unis (ibid., p. 90). Mais la fin du $\mathrm{XIX}^{\mathrm{e}}$ bouleverse l'économie et la démographie du pays. Des gisements ferrugineux sont découverts dans le sud du pays qui intensifient son activité industrielle et génèrent une demande accrue de main-d'œuvre trop rare au Luxembourg (Kreins, 1996, p. 83). On recourt à une abondante immigration, d'abord allemande puis italienne, pour faire face aux besoins toujours plus importants. Cet élan sidérurgique provoque un mouvement migratoire suffisamment important pour que le pays compte $12,3 \%$ de ressortissants étrangers en 1900, composés pour 25,6 \% d'Italiens, de Français pour 6,5\% et de Belges francophones pour 13,4\% (Trausch, 1992, p. 91). Le français se répand alors dans le bassin minier du sud $\mathrm{du}$ pays. Les Italiens s'en emparent par proximité avec leur langue. Ils peuvent ainsi communiquer avec les Français et les Belges. Mais le français reste cantonné aux échanges entre ouvriers, aux commerces de villages et il est surtout localisé à la seule région des mines, à la frontière actuelle avec la France et la Belgique. À la même époque, une revendication monte au sein de la population autochtone pour une reconnaissance de la variété allemande locale, le luxembourgeois. D'abord timide et circonscrite à quelques cercles (Trausch, 1992, p. 110), elle reprend de la vigueur après la Première Guerre mondiale, suite à l'annexion et à la germanisation du pays par la Prusse de 1914 à 1918 (Scuto, 2005, p. 13). L'idée d'un peuple qui n'est pas seulement bilingue allemand-français, mais trilingue luxembourgeois-allemand-français commence à germer dans le cœur de la population. L'affirmation se fera publiquement pendant la Seconde Guerre mondiale quand la population luxembourgeoise dans son ensemble, intégrée de force au III $^{\mathrm{e}}$ Reich, refusera de reconnaitre l'allemand comme langue maternelle, préférant revendiquer le luxembourgeois (Trausch, 1992, p. 169). Dans l'immédiate après-guerre, et bien que le législateur luxembourgeois s'abstienne d'entériner cette triglossie dans la réforme constitutionnelle de 1948, le luxembourgeois est désormais présent de facto. Sa reconnaissance de jure se réalise par la loi du 24 février 1984 sur le régime des langues ${ }^{2}$ qui dispose en son article premier que le luxembourgeois est «la langue nationale des Luxembourgeois ». L'article 2 rappelle que le français est la langue législative et réglementaire. L'article 3 garantit l'usage du luxembourgeois, du français et de l'allemand en matière administrative et judiciaire. Autrement dit, si le français n'a pas perdu sa position de prestige dans la législation, le luxembourgeois acquiert une existence légale, voire patrimoniale. De fait, la loi de 1984 se présente comme une mesure conservatoire de sauvegarde pour la variété indigène, perçue alors comme menacée de disparition. En effet, le Grand-Duché connait depuis le milieu des années 1960 une croissance économique 
vigoureuse qui provoque un nouveau besoin substantiel de main-d'œuvre étrangère (Trausch, 1992, p. 215). Le développement économique est soutenu par deux types de travailleurs étrangers : les étrangers très qualifiés qui travaillent pour le secteur bancaire et financier ou les institutions européennes installées dans le pays ; les étrangers peu qualifiés, majoritairement portugais, employés dans les secteurs de la construction et des infrastructures. Cette abondante main-d'œuvre lusophone en constante progression $-8 \%$ de la population totale en 1981 et $16 \%$ de la population totale en $2018^{3}$ - adopte le français comme langue d'intégration, renforçant ainsi sa fréquence dans le pays. Cette recrudescence du français parlé à partir de 1970 attire à son tour les populations frontalières françaises et belges, motivées par le niveau élevé de rémunérations. Et cette présence frontalière vient renforcer la diffusion du français à un point tel qu'il devient « la langue que le plus grand nombre de gens parlent et comprennent au Luxembourg » (Fehlen, 1998, p. 16). Le français assume, à la fin $d u X X^{\mathrm{e}}$ siècle, la position de première langue d'intégration au Grand-Duché dans un pays qui compte $48 \%$ de résidents étrangers au 31 décembre $2017^{4}$. Selon une enquête menée en 2008 par le sociolinguistique luxembourgeois Fernand Fehlen, le français est la langue « la mieux parlée par l'ensemble de la population », soit $96 \%$ de tous les résidents, devant le luxembourgeois (78\% des résidents), l'allemand (75\% des résidents) et l'anglais (61\% des résidents) (Fehlen, 2009, p. 80). Par ailleurs, le français est la première langue parlée au travail au Luxembourg (Heinz et Fehlen, 2016, p. 2). Enfin, il est plus largement maitrisé dans la population autochtone du fait de la démocratisation de l'accès à l'enseignement secondaire dans lequel il est langue d'enseignement de la filière classique, filière la plus valorisée au Grand-Duché (Esmein, 1998, p. 57).

Ce qu'il gagne en expansion, le français le perd en prestige. Langue du Luxembourg la plus apprise depuis 1970, sorte de koinè intercommunautaire du pays, le français souffre auprès des Luxembourgeois de son appropriation par les immigrés actifs dans les secteurs requérant le moins de qualifications. Par ailleurs, il est associé aux travailleurs frontaliers perçus comme des «monolingues arrogants qui ne font pas l'effort de s'approprier des notions minimales de luxembourgeois » (Conseil de l'Europe, 2005, p. 13). Enfin, l'élargissement de sa diffusion au sein de la majeure partie des résidents étrangers a suscité une réaction populaire traduite politiquement par la loi du 23 octobre 2008 et l'adoption d'un code de la nationalité rendant l'apprentissage du seul luxembourgeois obligatoire pour obtenir la naturalisation (Scuto, 2010, p. 94). Auparavant, la maitrise du français ou de l'allemand était suffisante pour satisfaire aux critères linguistiques d'intégration du pays. Ces deux langues sont d'ailleurs sur le point de perdre le statut d'officialité. En effet, le texte d'une réforme constitutionnelle adopté le 21 mars 2018 à l'unanimité des députés prévoit, en son article 4, que seul « le luxembourgeois sera la langue du Luxembourg ${ }^{5} »$. 


\section{Collecte de témoignages}

Une série de témoignages vient baliser le fil rouge de la présente communication. Ils ont été collectés auprès de Luxembourgeois natifs lors de la rédaction d'un mémoire de Master de recherche portant sur les représentations du français dans la triglossie luxembourgeoise en 2014 d'une part ; à l'occasion de la rédaction d'une thèse de doctorat traitant des politiques linguistiques d'intégration au Luxembourg entre 2015 et 2017 d'autre part. Ils émanent respectivement d'un appel à témoin (la condition préalable étaient qu'ils soient âgés de 16 ans au minimum) et d'une prise de contact directe auprès de professionnels (enseignants, animateurs associatifs, assistants sociaux, éducateurs), impliqués dans la mise en place concrète des politiques linguistiques locales. Les deux séries d'entretiens ont été réalisées sur le mode semi-directif ou interactif tel que défini par Jacques Bres (Bres, 1999, p. 68), sur base d'une série de questions établies préalablement et soumises aux informateurs témoins. Des extraits de ce corpus sont insérés ci-après pour rendre compte de la perception des variations linguistiques dans le chef des locuteurs endogènes.

Ainsi, le témoignage de Fr., Luxembourgeoise native de 42 ans mariée à un Luxembourgeois natif, illustre les changements simultanés de fréquence et de statuts du français entre la fin de la Seconde Guerre mondiale, où il était encore une variété rare et prestigieuse, et l'époque actuelle où il a perdu de sa valeur symbolique.

13 - I : Tu es native du Nord du Sud ou du Centre del

$14-\mathrm{Fr}: / \mathrm{Du}$ Centre

15 - I : Du Centre donc y'a quand même une bonne pratique du français dans le centre de Luxembourg

16 - Fr : Oui mais quand moi j'ai grandi... y'avait pas encore tant de Français dans les magasins que maintenant hein... et y'avait encore beaucoup plus de vendeuses qui parlaient l'luxembourgeois donc j'ai grandi dans un ... dans un

17 - I : Un environnement

18 - Fr : Un environnement où on parlait beaucoup plus luxembourgeois que maintenant

19 - I : Alors t'as le sentiment qu'il y a de plus en plus de francophones dans le pays ?

$20-\mathrm{Fr}: \mathrm{Oui}$

21 - I: Ouais?

22 - Fr: Ah oui... tout à fait... parce que je me rappelle quand j'étais plus jeune... j'entrais dans un magasin... aujourd'hui je sais... toutes les vendeuses vendeurs vont parler français et à l'époque c'était pas encore si heu... si prononcé

(...)

51 - I : Tu trouves que le français... a pour les Luxembourgeois ou au Luxembourg une réputation de... langue valorisante?

52 - Fr: A l'époque oui maintenant je ne sais pas mais à l'époque comme c'était pas la langue parlée de tous les jours mais c'était si on voulait faire quelque chose d'officiel... on l'écrivait en français... donc c'était toujours une langue comme moi je l'ai vécu dans ma famille...c'était pas langue de tous les jours pas la langue 
parlée pas la langue du cœur ...mais la langue si on voulait exprimer quelque chose très heu... très correctement ou officiellement alors on utilisait le français

53 - I : Et c'était pas l'allemand?

54 - Fr : L'allemand je m'rappelle que pour mes grands-parents s'ils écrivaient une lettre c'était en allemand...mais avec la deuxième guerre mondiale ça a changé parce que les nazis voulaient imposer l'allemand... donc c'était ou on a beaucoup heu... j'ai un grand-père qui était ... enrôlé de forcel

55 - I : /Un malgré-nous/

56 - Fr : / Ou d'autres qui étaient en camp de concentration donc c'était... l'allemand ça ne se voyait plus bien de parler l'allemand parce que ça leur donnait des souvenirs... mais pour les luxembourgeois l'allemand c'est plus naturel hein... parce que c'est beaucoup plus proche bien sûr

\section{Pluriglossie dynamique et variations diastratiques}

Pour Henri Boyer, l'analyse des diglossies « requiert une perspective historique et une prise en considération non seulement des usages des langues en présence dans une société mais également des représentations, des attitudes susceptibles de peser sur la dynamique de ces usages, dans le cadre de situations conflictuelles, de rapports de dominance entre les langues en présence »(Boyer, 2001, p. 18). Les pluriglossies sont donc avant tout diachroniquement instables et dynamiques. La situation luxembourgeoise ne déroge pas à la règle.

À son avènement définitif en 1839, la situation sociolinguistique du pays s'apparente au modèle élaboré par Joshua A. Fishman de «diglossie avec bilinguisme ». Cette structure décrit un environnement dans lequel deux variétés coexistent sur un même territoire - le cas échéant le français et l'allemand - et sont pratiquées par une part substantielle de la population locale (Fishman, 1972, p. 75). Certes, en 1839, seule la grande bourgeoisie maitrise le français mais, étant l'une des deux langues législatives de l'époque, il est censé être accessible au plus grand nombre. L'émergence d'une revendication de la variété germanique locale, l'allemand luxembourgeois bientôt émancipé de son patronyme « allemand » à partir de 1920, fait glisser la diglossie luxembourgeoise vers une pluriglossie enchâssée (Calvet, 1999, p. 47) à l'intérieur de laquelle s'imbrique le modèle de Fishman précité, entre le français et l'allemand, et celui de Charles A. Ferguson. Ce dernier conçoit un système diglossique dans lequel cohabitent deux variétés d'une même langue, chacune possédant une «spécialisation de fonction » (Ferguson, 1959, 235) : une variété littéraire, protocolaire et cérémoniale dite « haute» $(\mathrm{H})$; une variété populaire et vernaculaire dite «basse »(B) (ibid., p. 236). La pluriglossie «enchâssée » du Luxembourg fait donc apparaître, au cours de la première moitié du $\mathrm{XX}^{\mathrm{e}}$ siècle, une architecture pyramidale agençant le français en son sommet - variété haute de prestige -, en son centre l'allemand, variété haute du diptyque germanique, et à sa base sa variante basse locale et vernaculaire, le luxembourgeois (Trausch, 1998, p. 21). Cette configuration est toutefois courante dans la sphère linguistique allemande où la variété standard haut allemand (Hochdeutsch) se confronte généralement à une variété dialectale régionale (Trabant, 2007, p. 73). Le sociolinguiste Fernand Fehlen rapproche la diglossie allemand/luxembourgeois de celle observée en Suisse alémanique (Fehlen, 2009, p. 43) et que le linguiste suisse Jakob Wüest 
nomme diglossie « médiale » pour décrire la relation entre l'allemand standard Hochdeutsch et les dialectes alémaniques (Wüest, 1993, p. 171). Suivant cette configuration, les variétés en coexistence n'entretiennent pas de relation de concurrence de prestige mais assument les fonctions de « code écrit » et « code oral » (ibid.) au sein d'une diglossie « neutre » dans laquelle « chaque langue possède des domaines qui lui sont propres " (ibid.). Cependant, Fehlen note que le contexte suisse se démarque du luxembourgeois en cela qu'au Luxembourg l'allemand standard est, d'une part, vécu comme une langue étrangère ; qu'il y assure d'autre part des fonctions spécifiques (langue de télévision, des médias, des journaux) alors qu'en Suisse, l'allemand standard n'est pas ressenti comme une variété exogène ni fonctionnellement marquée (Fehlen, 2009, p. 44). Cette distribution diglossique des deux langues persiste encore actuellement comme le démontre le témoin C., Luxembourgeoise native de 50 ans mariée à un Luxembourgeois natif, dans sa relation avec l'allemand :
$37-I$ : L'allemand tu l'utilises presque jamais/
$38-C:$ Presque jamais
39 - I : Presque jamais... sauf à la télé peut-êtrel
$40-C$ : IJe lis des bouquins allemands... j'regarde la télé allemande... je... tout ça
on comprend l'allemand comme si c'serait notre heu... on comprend tout... et ça va jamais être le même cas pour le français... ça va jamais être pareil... l'allemand c'est trop proche du luxembourgeois... on comprend... et donc si on parle... du coup si on parle allemand on parle une mixture luxembourgeois allemand... tu vois... et c'est trop moche après... c'est super moche
$41-I$ : C'est important pour toi l'esthétique de la languel
$42-C$ : /Oui mais les Allemands y sont là comme ça... y rigolent mais bon... c'est gênant

La dynamique pluriglossique mène, au début du XXI ${ }^{\mathrm{e}}$ siècle, le Luxembourg vers une nouvelle configuration dans laquelle le luxembourgeois tient lieu de variété « nationale »patrimoine des détenteurs de la nationalité luxembourgeoise -, variété haute et de prestige ; l'allemand standard se maintient en tant que corollaire scripturaire et, à ce jour, langue scolaire d'alphabétisation ${ }^{6}$; le français constitue désormais la base de cette structure, étant à la fois langue d'accès des migrants et des frontaliers à l'économie et aux administrations du pays, et langue des professions les moins qualifiées (Langers, 2010, p. 254). Les migrants hautement qualifiés se caractérisent par leur pratique quasi exclusive de l'anglais (Hartmann-Hirsch, 2010, p. 127).

Si les architectures pluriglossiques sont instables, c'est notamment parce que les langues qui les composent sont parfois amenées à changer de statut en fonction des locuteurs qui se les approprient dans l'espace social. On parle alors de leur variation diastratique. Cette variation, définie par le romaniste norvégien Leiv Flydal, se propose de mesurer la position de la langue donnée dans la hiérarchie sociale (Glessgen, 2012, p. 111), composée de différentes couches ou strates. Le concept de variation diastratique a été reformulé par le linguiste roumain Eugenio Coseriu pour rendre compte des «variations naturelles de la 
parole créée par l'individu » en fonction de paramètres qui les conditionnent : les variations dans le temps (la diachronie), dans l'espace (la diatopie), dans la société (la diastratie), dans le choix du registre de langue (la diaphasie), ou même de la situation ou du support de l'énonciation (la diamésie) (Van Eyck, 2009, p. 7). Le principe fondamental de ces variations est « qu'il n'est pas de langue que ses locuteurs ne manient sous des formes diversifiées » (Ledegen \& Léglise, 2013, p. 315). Le concept de «variation » désigne les différenciations de l'énonciation en situation de synchronie, c'est-à-dire, compte tenu du contexte contemporain de cette énonciation. Les variations diastratiques tiennent donc compte des caractéristiques sociales des locuteurs (ibid., p. 316).

Dans la structure sociolinguistique luxembourgeoise, le français a connu des variations diastratiques qui l'ont mené du statut de langue de prestige à celui de langue dévalorisée des étrangers parce qu'il a changé de communauté de locuteurs. D'abord chasse gardée d'une haute bourgeoisie cultivée mais jalouse de ses prérogatives au sommet de l'État, il représente longtemps un vecteur de l'élévation sociale de ceux qui ont pu suivre une scolarisation longue. À ce titre, son manque de maitrise par les Luxembourgeois a pu représenter une preuve stigmatisante d'un manque d'éducation comme le rappelle le témoin C. :

$78-C$ : Le problème c'est qu'y a beaucoup de Luxembourgeois qui ont des problèmes avec le français... c'est pas que... y sont pas gênés de parler devant des Français... de faire des fautes mais y sont gênés de parler devant des Luxembourgeois et de faire des fautes... parce qu'au Luxembourg... si tu parles pas bien l'français t'es vu comme quelqu'un qui n'a pas fait d'études... donc y critiquent... disons si t'es quelqu'un de éduqué... et que t'as fait ton unif... université... t'étais à Paris ou t'étais j'sais pas où... en Belgique au moins.... et donc tu sais... bien parler heu... et si tu ... c'est encore vu comme ça... c'est beaucoup vu comme ça... et c'est c'que va te dire beaucoup de Luxembourgeois... les gens sont intimidés vis-à-vis des Luxembourgeois parce que y en a trop qui l'parlent super bien... et si tu parles pas bien t'es toujours vu comme quelqu'un qui n'a pas fait d'études... t'sais c'qui y a le problème aussi avec les Luxembourgeois qui font leurs études en Allemagne ou à Berlin y reviennent et l'français est un peu heu... y savent plus trop bien l'utiliser ça pose un très grand problème... y a bien sûr des Luxembourgeois qui ne l'utilisent jamais... mon mari il l'utilise tous les jours au boulot... heu...moi aussi donc... mais y a des Luxembourgeois qui l'parlent jamais... qui n'en ont pas besoin

Ce témoignage fait écho à «l'hypercorrection crispée » que relève F. Fehlen à propos de l'utilisation du français par les Luxembourgeois (Fehlen, 1998, p. 17). Mais il note aussi qu'il existe deux perceptions du français dans la société. «Le français qu'on parle en haut et celui qu'on parle en bas de l'échelle n'ont pas les mêmes valeurs socioculturelles : d'un côté, on rencontre l'emploi d'un français cultivé et de l'autre, il s'agit de ce qui pourrait être appelé un " pidgin" franco-portugais-luxembourgeois. " (ibid.). Ces deux formes de français font référence à deux types distincts de son acquisition. Le premier type renvoie à l'apprentissage du français par les Luxembourgeois natifs à travers le système scolaire local. Il est alors une langue-matière abordée de manière théorique et distanciée par de jeunes élèves déjà confrontés à une diglossie pédagogique entre le luxembourgeois parlé et 
l'allemand standard écrit. A.E., Belge et enseignante installée au Luxembourg depuis 1969, rend compte de la difficulté éprouvée par les élèves vis-à-vis du français :

94/ A. E. : Alors c'est pas nouveau... les jeunes Luxembourgeois aiment pas le français... heu... à mon idée c'est un problème que je connais bien on peut en discuter... heu la première raison c'est que pour eux on leur explique pas heu... comment dirais-je... la notion historique du français à Luxembourg... pour eux le français n'est pas un moyen de communication... c'est une matière scolaire... qui... maintenant c'est mieux mais qui était au départ extrêmement... pas mal enseignée mais...enseignée de manière très rébarbative... les petits Luxembourgeois sont incollables sur la grammaire française...mais... pour parler... c'est compliqué... moi j'ai eu en cours privé des petits Luxembourgeois qui disaient j'aime pas le français** parce que chez eux... heu...on écoute la télé en allemand...ou en anglais... l'anglais c'est la langue heu...musique et tout ça... si on va au cinéma on prend les films américains... donc le contact qu'ils ont avec le français n'est qu'un contact scolaire... pas très... heu... joyeux... parce que faire tout un cours sur les pronoms relatifs heu... voilà

Le deuxième type d'acquisition est celui opéré par les migrants romanophones qui se sont emparés du français par contact et par mimétisme, "sur le tas », sans prendre de cours et pour satisfaire aux nécessités quotidiennes et aux contraintes administratives et professionnelles locales, mais qui ne l'utilisent que pour les besoins d'échanges systématiques et circonscrits aux cercles extra-familiaux et extra-communautaires.

\section{Sociolecte et marché linguistique}

La diffusion du français, à la fin $\mathrm{du} \mathrm{XX}^{\mathrm{e}}$ siècle, a principalement été le résultat de « l'immigration portugaise qui, en développant l'usage véhiculaire du français dans le pays, a rendu possible dans un deuxième temps les mouvements transfrontaliers amenant les francophones à utiliser assez massivement cette nouvelle ouverture linguistique » (Esmein, 1998, p. 67). Le français est alors devenu le véhicule d'une communauté ciblée, les Portugais, dans un premier temps ; les frontaliers dans un second temps. Il arbore depuis les attributs d'un sociolecte, autrement dit d'indicateur d'appartenance à « des couches ou des groupes sociaux spécifiques »(Glessgen, 2012, p. 113). En effet, dans la sociolinguistique luxembourgeoise, le français jouit d'une valorisation à condition qu'il soit la composante d'une compétence nationale légitime qui inclut également la maîtrise du luxembourgeois et de l'allemand. Hors de cette triple compétence, le français n'est plus que le vecteur minimal d'entrée dans la société luxembourgeoise mais il est réduit à un simple rôle d'intégrateur. Il n'est plus un marqueur spécifique d'élévation intellectuelle et sociale tel qu'il peut l'être au sein de la communauté nationale.

À l'image d'un produit doté d'une valeur d'échange et d'une valeur d'usage, à l'intérieur d'un «marché linguistique » tel que l'a défini le sociologue Pierre Bourdieu (Bourdieu, 1984, p. 124), la cote de la langue française a varié en fonction de sa rareté et de la couche sociale qui se l'appropriait. Variété exclusivement utilisée par les lettrés et les politiciens pendant plus d'un siècle, il bénéficiait d'une valorisation très élevée sur le marché linguistique luxembourgeois. « D’un autre côté, une langue ne devient pas la langue 
que l'on parle avec les vendeurs, les femmes de ménage et les serveurs de restaurant sans que son image symbolique n'en soit affectée » (Esmein, 1998, p. 57). L'accroissement de sa fréquence et de son emploi dans toutes les couches de la société, notamment les plus basses, a eu pour effet de dévaluer le français au point de la confiner au rang de sociolecte des étrangers, par opposition à ceux qui connaissent le luxembourgeois.

Dans la logique développée par Bourdieu, un marché linguistique est un espace dans lequel un discours produit par un locuteur est évalué et estimé par les récepteurs de ce discours et qui met en scène un certain nombre d'acteurs et de mécanismes à même de conférer un prix à cette production linguistique. L'idée de marché implique nécessairement des tensions et des rapports de force propres à apprécier et valoriser les discours. De ce fait, un marché sous-tend également des acteurs régulateurs, des détenteurs de capital linguistique, des producteurs, des acquéreurs-consommateurs à l'image d'un marché d'échange de biens de consommation quelconque. En prolongeant la métaphore, on peut substituer aux termes techniques de marché les notions linguistiques :

- les biens négociables et de consommation : les langues en présences dotées d'une considération plus ou moins élevée en contexte multilingue (le Luxembourg, par exemple) ; la langue dominante dans sa réalisation la plus gratifiée médiatiquement et socialement en situation monolingue (la France, par exemple);

- les détenteurs du capital linguistique : ceux qui détiennent une maîtrise jugée convenable des langues en présence valorisées ; ceux qui détiennent la compétence valorisée de la langue légitime ;

- le capital linguistique : le pouvoir sur les mécanismes des prix linguistiques et le pouvoir de manipuler les prix à son profit ;

- les régulateurs qui contrôlent la qualité de la production linguistique, la norme (Bourdieu, 2001, p. 70), et qui la promeuvent : les institutions administratives qui confèrent le statut de "langue officielle » ou "langue nationale », l'école, les médias, les cénacles autorisés ;

- les producteurs, acquéreurs, consommateurs : usagers, locuteurs détenteurs ou non de la ou des compétences légitimes.

Le système de Bourdieu implique deux préalables : un marché unifié et identifié par tous ainsi que la reconnaissance implicite d'une autorité qui tranche entre la légitimité et l'illégitimité de la pratique langagière. Le marché unifié est principalement associé à des limites territoriales, donc aux frontières de l'État. L'autorité implicite procède de l'histoire politique, institutionnelle et culturelle de l'État (Bourdieu, 1984, p. 124).

Sa grille peut se transposer au contexte multilingue du Grand-Duché de Luxembourg où la compétence légitime a d'abord été délimitée par la communauté autochtone des Luxembourgeois pour enfin être entérinée par la loi du 24 février 1984 sur le régime des langues. En l'absence de réel référent littéraire et académique, le luxembourgeois s'impose comme légitime parce qu'il est la langue de la communauté des natifs et que cette langue ne s'enseignait pas (compte tenu de son absence d'existence légale avant 1984) mais se transmettait de membre à membre de la communauté, par la famille d'abord, puis par 
l'entourage social. Les langues officielles depuis l'indépendance du pays en 1830 étant le français et l'allemand, la compétence légitime est donc l'usage indifférencié de ces trois langues historiques du pays (Esmein, 1998, p. 44). Sur un marché qui se diversifie à partir de la deuxième moitié du $\mathrm{XX}^{\mathrm{e}}$ siècle, la multiplication des langues sur le territoire (avec la présence notable de l'italien, du portugais puis de l'anglais) ajoute une forte plus-value au luxembourgeois, contenu à un cercle fermé qui est seul détenteur de ce capital, assorti de la double compétence allemand-français. Le français n'est plus l'élément saillant de l'éducation mais un trait éventuellement symptomatique de déclassement lorsqu'il est la seule variété indigène connue au détriment des deux autres. Comme le rappelle le témoin C., le français est la compétence minimale parce que tout le monde le parle :

43 - I : Est-ce que pour toi... d'après toi le français c'est... important ou nécessaire
à Luxembourg/
$44-C$ : IAh oui
$45-I$ : Oui... pourquoi ?
$46-C$ : Ben parce que tout l'monde le parle... déjà tout l'monde le parle déjà c'est
... c'est la langue heu... administrative... c'est la première langue administrative
j'dirais même... surtout en ville... Luxembourg-ville y'a tellement de frontaliers...
y'a tellement de gens de partout c'est tellement cosmopolite... que c'est toujours la
langue heu... que tout l'monde heu... même s'ils sont pas français... on discute
entre nous... on parle français en fait

\section{Langue et identité nationale}

La dévaluation du français est enfin le produit de son exclusion du champ des critères de définition de l'identité luxembourgeoise par les autochtones. Par la loi du 24 février 1984 sur le régime des langues et la loi du 16 décembre 2008 sur l'accueil et l'intégration des étrangers au Grand-Duché de Luxembourg ${ }^{7}$, la langue luxembourgeoise est devenue la seule variété linguistique du pays reconnue comme inhérente à la communauté nationale et pourvoyeuse de la nationalité luxembourgeoise. Dans le souci de se constituer en nation, le Luxembourg érige la langue luxembourgeoise en patrimoine linguistique endémique et historique qui lui permet de différencier les membres de la communauté nationale de ceux des autres communautés parce que ce patrimoine se transmet de génération en génération mais ne s'enseigne pas, au contraire des langues françaises et allemandes qui sont apprises dans le multilinguisme local (Frisch, 1998, p. 137). Le français - ni l'allemand a fortiori n'appartient plus au catalogue des traits culturels distinctifs et mutuellement partagés qui légitiment la spécificité du groupe, et donc de son identité. Adopté par l'intégralité des migrants d'une part, langue nationale de deux grands pays frontaliers - la France et la Belgique - d'autre part, la langue française est exclue des éléments constitutifs de l'historiographie nationale (Lagarde, 2008, p. 56). Comme le souligne Sarah Vasco dans son étude de 2011 sur la communauté portugaise du Luxembourg, la grande fierté nationale des Luxembourgeois repose sur son multilinguisme, mais un multilinguisme dans lequel la langue luxembourgeoise détient une position dominante (Vasco, 2011, p. 98). Plus encore, le multilinguisme national n'a de valorisation que s'il implique le luxembourgeois dans le 
répertoire verbal des locuteurs. La présence du seul français ou du seul allemand (encore entendus comme variétés institutionnelles du pays) dans le plurilinguisme des étrangers ne bénéficie pas d'une appréciation équivalente : «Il est certain que l'on peut vivre au Luxembourg sans maîtriser un mot de la langue nationale, cependant, semblent s'amoindrir les opportunités d'une trajectoire socioprofessionnelle ascendante et d'une insertion dans le noyau dur de la société luxembourgeoise » (ibid.).

Dans ce nouveau paradigme de pluriglossie nationale, le français, variété apprise, est perçu comme une langue étrangère que les natifs peuvent connaître mais ne doivent pas obligatoirement maîtriser avec aisance. S'il est encore le support des communications institutionnelles, il n'est pas le véhicule de communication de la communauté nationale. Les natifs peuvent donc affecter un manque de compétence dans sa pratique parce que c'est une langue finalement étrangère dont la possession approximative n'est plus préjudiciable. Les propos du témoin S., Luxembourgeoise native, enseignante au niveau primaire, sont explicites quant au confinement du français à une sphère fonctionnelle d'échange avec les travailleurs frontaliers sans nécessité de compétence particulièrement avancée :

162/ I : Mais on pourrait penser que les Luxembourgeois ont un complexe d'infériorité vis-à-vis de leur pratique du français ou de l'allemand parce qu'on peut comparer avec des natifs... tandis que le luxembourgeois... ils sont les seuls à le connaître... à le pratiquer... et là on peut pas comparer

163/ $S$ : C'est aussi une question d'identité

164/ I : Oui ça c'est sûr... une langue c'est un drapeau de l'identité

165/ S : Oui... mais ce que je voulais dire aussi par posséder... tu vois quand je parle français... heu... ça me vient pas tout seul

166/I : Ah oui... et toi t'es frontalière

167/ S : Oui si tu veux... et moi j'ai été mariée avec un Français alors... et j'ai fait les études en France... mais ça reste... je trouve pas le mot... je dirais étranger mais étranger c'est exagéré... c'est pas aussi fort que ça mais ça reste un petit peu étranger... l'allemand aussi... le luxembourgeois pas du tout... le luxembourgeois c'est ma langue de naissance... ma langue maternelle

168/I : Tes parents étaient tous les deux luxembourgophones

169/S: Oui

170/I: Toi tu es frontalière?

171/S: Oui

172/ I : Tu habites ici donc tu fais tes courses dans le coin?

173/ S:Oui

174/ I : Les employés des supermarchés des stations-service... des choses comme ça c'est des Belges

175/ S: Oui et des Français aussi

176/I: Donc tu es très souvent confrontée au français depuis que tu es née en fait

177/S : Oui mais dans le temps c'était moins

178/I : C'est vrai?

179/ S: Oui dans le temps on avait encore des petites épiceries et tout ça... c'était encore en luxembourgeois... oui moi je suis déjà la génération... tu vois... c'était pas comme ça à l'époque... moi quand j'étais à l'école primaire heu... on a eu un 
élève portugais dans ma classe... c'est la première fois que j'ai eu un contact heu... et ça m'a fascinée... je me souviens ça m'a fasciné... waoh ... il parle portugais... et on a été inondés par la télé allemande... et les livres... dès le début tout a été en allemand parce que... y avait pas encore des livres en luxembourgeois ou très très peu... et le français ça c'est venu un peu plus tard

\section{Conclusion}

Christian Lagarde cite le linguiste catalan Lluís Vicent Aracil dans une prophétie concernant les environnements plurilingues :

« Dans une société donnée, le bilinguisme n'est guère qu'une phase transitoire d'un monolinguisme à l'autre. Il en résulte la hantise de la substitution linguistique, considérée comme un processus inéluctable qui impliquerait l'individu, constamment mis en demeure de choisir son camp, sommé de réintégrer les rangs de sa tribu ou de se situer dans l'autre" " (Lagarde, 2008, p. 63).

Au Luxembourg, la revendication du trilinguisme national est en train de s'effacer au profit de la valorisation du monolinguisme luxembourgophone, caractère irréductible de l'appartenance à la communauté nationale. Cette évolution procède d'une crainte de la dilution, voire de la disparition de la langue luxembourgeoise, dans une société composée d'étrangers pour près de $50 \%$ de la population. Cette préoccupation a donné lieu à des réactions sociales, relayées politiquement par une modification du code de nationalité exigeant la compétence du seul luxembourgeois comme critère linguistique d'intégration en 2008 (alors que la maîtrise du français ou de l'allemand étaient suffisants avant cette réforme (Scuto, 2010, p. 92) ; un refus des étrangers de participer aux élections nationales lors d'un référendum organisé le 7 juin $2015^{8}$; une pétition publique déposée le 16 août 2016 auprès de la Chambre des députés, enregistrée sous le numéro $698^{9}$, demandant que le luxembourgeois devienne la première langue nationale du pays avant l'allemand et le français.

L'ultime variation diastratique du français dans l'environnement sociolinguistique luxembourgeois est dès lors son exclusion du patrimoine linguistique du pays. Variété présente dans le paysage national, le français risque de perdre sa légitimité officielle, puis, à terme, sa fonction intégrative. Le sociolecte français a évolué en fonction de sa position au sein de l'environnement luxembourgeois. De sociolecte interne assurant une distinction [classe supérieure-classe populaire], il a été extrait du champ linguistique de la " nation» pour servir de séparateur [national-étranger]. Ses variations n'ont pas tant reflété une démocratisation de son apprentissage qu'un désinvestissement complet des Luxembourgeois devant son adoption par les migrants. Le français n'est donc progressivement plus une langue du Luxembourg, mais une langue parlée au Luxembourg. Il subit en outre, à l'instar de l'allemand (Hochdeutsch), la concurrence de l'anglais, langue véhiculaire des fonctionnaires des institutions européennes sises au Luxembourg, ainsi que des employés du secteur bancaire et financier, pan important de l'économie locale $(11 \%$ 
des emplois totaux du pays ${ }^{10}$ ). L'expansion de cette langue, dispensatrice au Luxembourg d'une image gratifiante de locuteur hautement qualifié, bénéficie de la double dynamique décrite par Mario d'Angelo dans sa description du phénomène de "glocalisation »: une réduction des coûts du pluralisme linguistique par la réduction à une seule langue de travail pour toutes les organisations internationales et scientifiques (d'Angelo, 2019, p. 71) ; une surabondance de productions culturelles en anglais de par l'étendue du marché potentiel (ibid., p. 72).

Une nouvelle forme de diglossie émerge donc au Luxembourg, qui s'appuie d'une part sur le luxembourgeois en tant que marqueur de l'appartenance à la communauté nationale, d'autre part sur l'anglais, vecteur d'ascension professionnelle.

\section{Notes}

${ }^{1}$ http://legilux.public.lu/eli/etat/leg/argd/1834/02/22/n1/jo

2 http://legilux.public.lu/eli/etat/leg/loi/1984/02/24/n1/jo

${ }^{3}$ https://statistiques.public.lu/stat/TableViewer/tableView.aspx

4 https://statistiques.public.lu/stat/TableViewer/tableView.aspx

5 http://www.land.lu/page/news/125/FRE/index.html

${ }^{6} \mathrm{http}: / /$ www.men.public.lu/fr/themes-transversaux/langues-ecole luxembourgeoise/index.html

${ }^{7}$ http://legilux.public.lu/eli/etat/leg/loi/2008/12/16/n5/jo

${ }_{9}^{8}$ https://gouvernement.lu/fr/actualites/toutes_actualites/articles/2015/06-juin/05-referendum-luxembourg.html

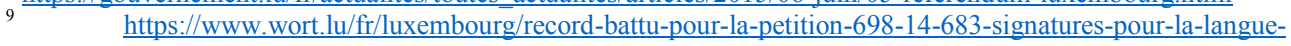
luxembourgeoise-580e0f675061e01abe83ae11

${ }^{10}$ https://statistiques.public.lu/stat/TableViewer/tableView.aspx?Reportld=12914\&IF Language=fra\&MainThem $\underline{\mathrm{e}=2 \& \text { FIdrName}=3 \& \text { RFPath }=92}$

\section{Bibliographie}

Berg C., Weis C., 2005, Sociologie de l'enseignement des langues dans un environnement multilingue, Rapport national en vue de l'élaboration du profil des politiques linguistiques éducatives luxembourgeoises, Ministère de l'Éducation nationale et de la formation professionnelle et Centre d'études sur la situation des jeunes en Europe.

Bourdieu P., 2001, Langage et pouvoir symbolique, Paris, Éditions Fayard / Éditions du Seuil, collection Points.

Bourdieu P., 1984/2002, Questions de sociologie, Paris, Les Éditions de Minuit.

Boyer H., 2001, Introduction à la sociolinguistique, Paris, Dunod.

Bres Jacques, 1999, «L'entretien et ses techniques », L'enquête sociolinguistique, Paris, L'Harmattan, p. 62-76.

Calvet L.-J., 1999, La guerre des langues et les politiques linguistiques, Paris, Hachette Littératures.

Conseil de l'Europe - Ministère de l'Éducation nationale et de la Formation professionnelle du Luxembourg, 2006, "Profil de la politique linguistique éducative - Grand-Duché de Luxembourg », Division des Politiques linguistiques, Strasbourg.

D’Angelo M., 2019, «Les langues dans la glocalisation. Le cas du français », Cahiers du CEREN, $n^{\circ}$ 52 , p. 69 à 76 .

Esmein ., 1998, «L'évolution de la situation linguistique et culturelle au Luxembourg depuis 1975 », La situation de la langue française parmi les autres langues en usage au Grand-Duché de Luxembourg, Centre culturel français de Luxembourg, Centre d'études et de recherches 
européennes Robert Schuman, Université de Metz / U.F.R. Lettres et Sciences humaines Département communication., p. 56-98.

Fehlen F., 1998, Hors-série 1: Le sondage « Baleine » Une étude sociologique sur les trajectoires migratoires, les langues et la vie associative au Luxembourg. Centre de Recherche Public-Centre Universitaire, Cellule STADE sous l'égide du Ministère de la Famille, Commissariat du Gouvernement aux Etrangers. Recherche Etude Documentation (R.E.D.). Luxembourg. Ed : SESOPI Centre Intercommunautaire.

Fehlen F., 2009, «Baleine Bis » Une enquête sur un marché linguistique multilingue en profonde mutation / Luxemburgs Sprachenmarkt im Wandel. Recherche Etude Documentation $\mathrm{n}^{\circ} 12$, Luxembourg, Ed : SESOPI Centre Intercommunautaire.

Ferguson C. A., 1959, «"Diglossia”, Language and Social Structures », World, vol. 15, p. 325-40

Fishman J. A., 1972, Sociolinguistics, a brief introduction, Rowley, Newbury House Publishers.

Frisch J.-C., 1998, « Le Luxembourg, pays multilingue », La situation de la langue française parmi les autres langues en usage au Grand-Duché de Luxembourg, Centre culturel français de Luxembourg, Centre d'études et de recherches européennes Robert Schuman, Université de Metz / U.F.R. Lettres et Sciences humaines - Département communication., p. 107-113.

Glessgen M., 2012, Linguistique romane, domaine et méthodes en linguistique française et romane, Paris, ed. Armand Colin.

Hartmann-Hirsch C., 2010, «À propos de la transnationalisation du marché de l'emploi : est-elle "policy driven" ou "market driven"? ", ASTI 30 +, ed. ASTI, Luxembourg, p. 125-137.

Heinz A., Fehlen F., 2016, « Regards sur les langues au travail, n 11, STATEC », Institut national de la statistique et des études économiques.

Kreins J.-M., 1996, Histoire du Luxembourg, Paris, Presses Universitaires de France.

Lagarde C., 2008, Identité, langue et nation, qu'est-ce qui se joue avec les langues, Canet, Trabucaire.

Langers J., 2010, «Immigrés : haro sur les moins qualifiés ? », ASTI 30 +, ed. ASTI, Luxembourg, p. 248-261.

Ledegen G., Léglise I., 2013, «Variations et changements linguistiques », Sociolinguistique des langues en contact, ENS Éditions, p. 315-329.

Scuto D., 2005, «Qu'est-ce qu'un Luxembourgeois ? Histoire de la nationalité luxembourgeoise du Code Napoléon à nos jours : une histoire sous influence française, belge et allemande ", Conférence $d u \quad 3$ mars 2005, Université de Luxembourg. http://s1.emonsite.com/2009/08/20/37712162qu-es-ce-qu-un-luxembourgeois-pdf.pdf

Scuto D., 2010, « Comment devient-on Luxembourgeois. Deux siècles de réformes du droit de la nationalité », ASTI 30 +, ed. ASTI, Luxembourg, p. 80-95.

Trabant J., 2007, « De la langue allemande : un avenir lourd du passé », Le français aujourd'hui 2007/1, $n^{\circ} 156$, p. 69-78.

Trausch G., 1992, Histoire du Luxembourg, Paris, Hatier.

Trausch G., 1998, « La situation du français au Luxembourg : une prééminence précaire dans un pays d'expression trilingue ", La situation de la langue française parmi les autres langues en usage au Grand-Duché de Luxembourg, Centre culturel français de Luxembourg, Centre d'études et de recherches européennes Robert Schuman, Université de Metz / U.F.R. Lettres et Sciences humaines - Département communication, p. 20-32.

Van Eyck R., 2009, «Présentation », Travaux de linguistique, $n^{\circ}$ 59, De Boeck Supérieur, p. 7-11.

Vasco S., 2011, « La communauté portugaise au Luxembourg. Peut-on parler de continuum intergénérationnel de la pratique de la langue et de la culture d'origine ? », Lengas $n^{\circ} 70$, pp. 95112. 


\section{Convention de transcription des entretiens}
$\mathrm{I}$ : interviewer
$?$ : interrogation
/ : chevauchement
... : hésitations
** : rires 